\title{
Trastornos del sueño en el niño y adolescente en aislamiento social durante la pandemia COVID-19
}

\author{
Sleep disorders in children and adolescents in social isolation during the \\ COVID-19 pandemic
}

Distúrbios do sono em crianças e adolescentes em isolamento social durante a pandemia COVID-19

\author{
Daniel Leonardo Cruz Montesinos (ㅁ) ', María Andrea Linares Contreras (1) 1, Mignia Ziadet \\ Bermúdez (1) ${ }^{2}$, Janiny Ortiz Luna (1) ${ }^{2}$, Francisco Morán Rizzo (1) ${ }^{2}$, Nathaly Baldeón Estrada (ib) ${ }^{2}$
}

\begin{abstract}
1 Servicio de Neurología Pediátrica, Hospital General del Norte de Guayaquil, IESS, Los Ceibos, Guayaquil-Ecuador; ${ }^{2}$ Servicio de Pediatría. Hospital General del Norte de Guayaquil, IESS, Los Ceibos, Guayaquil-Ecuador.
\end{abstract}

\section{Correspondencia a:}

Daniel Leonardo Cruz Montesinos, dennis_cruz2002@yahoo.com

Recibido: 20 de mayo, 2021

Aceptado: 10 de septiembre, 2021

Publicado: 18 de noviembre, 2021

\section{ARTÍCULO ORIGINAL}

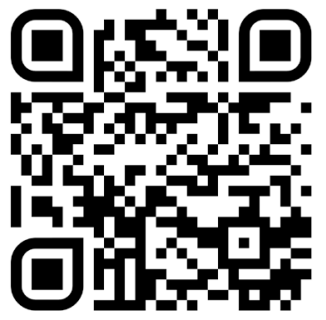

Escanea en tu dispositivo móvil o revisa este artículo en: https:// revistaclinicaguayaquil.org

\section{RESUMEN}

Introducción: La pandemia por coronavirus 2019 (COVID-19) es una emergencia de salud pública con impactos significativos en la salud mental de la población general. El aislamiento social y el confinamiento en el hogar pueden estar relacionados con el desarrollo de trastornos del sueño.

Materiales y Métodos: El presente estudio descriptivo prospectivo fue realizado durante los meses de febrero y marzo del 2021. Se aplicó una encuesta dirigida a identificar trastornos del sueño en 155 niños y adolescentes.

Resultados: La prevalencia de trastornos del sueño en niños y adolescentes fue del $35 \%$. Por medio de la escala de trastornos del sueño de Bruni se determinó tres principales trastornos del sueño: trastorno de inicio y mantenimiento del sueño (37\%), hiperhidrosis del sueño (20\%), somnolencia diurna excesiva (17\%).

Conclusiones: Si bien estos resultados no pueden ser extrapolados a la población general, la importancia del mismo radica en concientizar a la población sobre los trastornos del sueño en los niños y adolescentes.

Palabras clave: Trastornos extrínsecos del sueño; Niño; Adolescente; COVID-19; Aislamiento social

\begin{abstract}
Introduction: The 2019 coronavirus pandemic (COVID-19) is a public health emergency with significant impact on the mental health of the general population. Social isolation and home confinement can be linked to the development of sleep disorders.

Materials and Methods: The present descriptive prospective study was carried out from February until March 2021. A survey aimed at identifying sleep disorders in 155 children and adolescents was applied.

Results: The prevalence of sleep disorders in children and adolescents was 35\%. Using the Bruni Sleep Disorders Scale, three main sleep disorders were determined: sleep onset and maintenance disorder (37\%), sleep hyperhidrosis (20\%), excessive daytime sleepiness (17\%). Conclusions: Although these results cannot be extrapolated to the general population, its importance lies in making the population aware of sleep disorders in children and adolescents.
\end{abstract}

Key words: Extrinsic sleep disorders; Child; Adolescent; COVID-19; Social isolation 


\section{RESUMO}

Introdução: A pandemia de coronavírus de 2019 (COVID-19) é uma emergência de saúde pública com impactos significativos sobre a saúde mental da população em geral. $O$ isolamento social e o confinamento domiciliar podem estar associados ao desenvolvimento de distúrbios do sono.

Materiais e Métodos: $O$ presente estudo descritivo prospectivo foi realizado durante os meses de fevereiro e março de 2021. Foi realizada uma pesquisa para identificar distúrbios do sono em 155 crianças e adolescentes.

Resultados: A prevalência de distúrbios do sono em crianças e adolescentes foi de 35\%. Por meio da escala dos distúrbios do sono Bruni foram determinados três distúrbios principais do sono: início do sono e distúrbio de manutenção (37\%), hiperidrose do sono (20\%), sonolência diurna excessiva (17\%).

Conclusões: Embora estes resultados não possam ser extrapolados para a população em geral, a importância destes resultados está na conscientização dos distúrbios do sono em crianças e adolescentes.

Palavras-chave: Distúrbios extrínsecos do sono; Criança; Adolescente; COVID-19; Isolamento social

\section{INTRODUCCIÓN}

En marzo de 2020 la enfermedad por coronavirus 2019 (COVID-19), fue declarada una pandemia que afecta a innumerables personas con resultados potencialmente mortales; con el objetivo de contener y reducir la propagación del virus se aplicaron en todo el mundo medidas de aislamiento social y de confinamiento en el hogar $(1,2)$.

Se ha comentado en estudios recientes, que el aislamiento social y el confinamiento en el hogar podrían tener efectos negativos en los hábitos, dada la interrupción de los horarios de actividades por fuera de la casa, dentro de casa, y rutinas destinadas propiamente para el hogar (3). Estos cambios pueden afectar la cantidad de horas que se destina para dormir o permanecer tiempo en cama, e implican un deterioro de las asociaciones positivas entre el hogar, relajación, ocio y el sueño, conllevando a mayores niveles de estrés $(4,5)$.

En relación al sueño en el ser humano, se conoce que es un proceso fisiológico activo y cíclico que tiene efectos críticos en la salud. El sueño está implicado en el crecimiento, desarrollo, aprendizaje, memoria, eficiencia sináptica, regulación del comportamiento, emoción, fortalecimiento inmunológico y tiempo de limpieza de sustancias neurotóxicas (6). Ciertos factores como estilos de vida familiares disfuncionales, horarios de sueño sin restricciones, exposición prolongada a pantallas, el acceso limitado a las actividades al aire libre y las interacciones con pares, y el aumento del estrés, podrían contribuir a patrones de sueño poco saludables y por ende a trastornos del sueño (4).

Los trastornos del sueño siempre han sido un problema frecuente en la población pediátrica. Su prevalencia fluctúa entre el 20 al $45 \%$ y su etiología puede ser diversa, siendo la más común la mala higiene del sueño (7). En el año 1996, Bruni y cols, sugirieron pesquisar los trastornos del sueño en pediatría en seis áreas: inicio y mantenimiento del sueño, problemas respiratorios, desórdenes del arousal (trastornos frecuentes que afectan a los mecanismos del despertar que impiden el control voluntario de la actividad motora), alteraciones del tránsito vigilia-sueño, somnolencia excesiva e hiperhidrosis del sueño (8).

El objetivo del estudio es conocer los principales trastornos del sueño en niños y adolescentes en aislamiento social durante la pandemia por COVID-19. Es conocido que una detección temprana y abordaje oportuno de los trastornos del sueño en pediatría tiene un impacto positivo en la calidad de vida del niño y su familia.

Este es el primer estudio descriptivo y prospectivo en Ecuador que aborda los trastornos del sueño en niños y adolescentes en aislamiento social por la pandemia COVID-19.

\section{MATERIALES Y MÉTODOS}

Se realizó un estudio descriptivo y prospectivo, basados en una muestra no probabilística a conveniencia. Fueron incluidos niños sanos mayores de tres años y menores de quince años de edad en aislamiento social durante la pandemia por COVID-19, evaluados en el servicio de consulta externa pediátrica del Hospital General del Norte de Guayaquil, IESS Los Ceibos, durante los meses de febrero y marzo de 2021.

Previamente se contó con la autorización del departamento de docencia del Hospital General del Norte de Guayaquil, IESS Los Ceibos, y de los padres o tutores. Se excluyeron del estudio a niños y adolescentes con diagnóstico de trastornos del sueño antes del aislamiento social por la pandemia por COVID-19, patologías 
crónicas que pudieran afectar a la calidad del sueño (epilepsia, enfermedades oncológicas, renales, asma, autismo, trastornos del neurodesarrollo), pacientes con fármacos que alteran el inicio o mantenimiento del sueño, y pacientes que al momento de la evaluación no se encontraran con un adulto conviviente con conocimiento de las características del sueño del niño.

Los datos fueron recolectados por tres pediatras voluntarios capacitados en el tema, el instrumento utilizado fue una encuesta dirigida a los padres o tutores del niño, la que fue aplicada por telemedicina y en consulta externa presencial (Anexo 1).

Se aplicó una entrevista estructurada de selección que identificaba edad, género, y alteración del sueño durante el aislamiento social por la pandemia COVID-19. A los pacientes cuyos padres certificaron que presentan una alteración del sueño se aplicó la escala de trastornos del sueño para niños de Bruni (validada para su utilización en niños de 2 a 16 años). La escala de sueño del Dr. O. Bruni es de uso libre y no hay que pagar derechos por uso del instrumento, consta de 26 ítems valorados según una escala tipo Likert diseñada para pesquisar posibles trastornos del sueño en los últimos 6 meses en seis áreas: inicio y mantenimiento del sueño, problemas respiratorios, desórdenes del arousal, alteraciones del tránsito vigilia-sueño, somnolencia excesiva e hiperhidrosis del sueño (Anexo 2). A cada uno de los 26 ítems se les da un puntaje del 1 a 5 (mayor valor a mayor frecuencia del síntoma). Un puntaje igual o mayor a 39 puntos en el total del cuestionario hace sospechar la presencia de un trastorno global del sueño $(8,9)$.

Los datos obtenidos fueron ingresados a una base de datos en Microsoft Excel, y se realizó una estadística descriptiva, promedio, frecuencia, porcentaje y distribución según el grupo de edad asignado. Los resultados son presentados por grupo etario: preescolares de 3 a 5 años, escolares de 6 a 12 años y adolescentes de 13 a 15 años.

\section{RESULTADOS}

Se realizaron 155 encuestas, la participación en el estudio fue aceptada de manera verbal por el $100 \%$ de los padres. Los pacientes fueron clasificados por el grupo etario correspondiente: 34 preescolares, 80 escolares y 41 adolescentes.
El 35\% de los niños y adolescentes en aislamiento social por la pandemia por COVID-19, presentaron algún trastorno del sueño (55/155). La edad promedio de presentación de trastornos del sueño en niños y adolescentes en aislamiento social por la pandemia por COVID-19 fue de ocho años, el valor de la moda fue de siete años, predominando en el género masculino. En los niños y adolescentes en aislamiento social por la pandemia por COVID-19, los trastornos del sueño por grupo etario fueron más frecuentes en los escolares $45 \%$, seguido por los preescolares $29 \%$ y adolescentes $25 \%$ (Tabla 1 ).

Tabla 1. Trastornos del sueño por grupo etario y género en el niño y adolescente en aislamiento social durante la pandemia COVID-19, Hospital General del Norte de Guayaquil, IESS, Los Ceibos, 2021.

\begin{tabular}{lccc} 
Grupo etario & $\begin{array}{c}\text { Femenino } \\
\mathbf{n = 2 3}\end{array}$ & $\begin{array}{c}\text { Masculino } \\
\mathbf{n = 2 2}\end{array}$ & $\begin{array}{c}\text { Total } \\
\mathbf{n = 5 5}\end{array}$ \\
\hline Preescolar & 6 & 10 & $16(29 \%)$ \\
Escolar & 13 & 12 & $25(45 \%)$ \\
Adolescente & 4 & 10 & $14(25 \%)$
\end{tabular}

Elaboración: Cruz D. 2021. Fuente: Formulario de recolección de datos.

En el grupo etario escolar el trastorno en el inicio y mantenimiento del sueño fue el más frecuente ( $52 \%$ de los casos), seguido de la hiperhidrosis $(28 \%)$, somnolencia diurna excesiva (24\%), trastornos en la transición sueño-vigilia $20 \%$, trastornos respiratorios $12 \%$, y trastornos del arousal 4\%. En el grupo etario preescolar, el trastorno en el inicio y mantenimiento del sueño fue el más frecuente (43\%), seguido de la hiperhidrosis (31\%), los trastornos de la transición sueño-vigilia (31\%), somnolencia diurna excesiva $(12 \%)$, y los trastornos respiratorios (6\%). En el grupo etario adolescente, el trastorno en el inicio y mantenimiento del sueño el insomnio fue el más frecuente (42\%), seguido de la somnolencia diurna excesiva (28\%), hiperhidrosis (14\%), trastornos respiratorios y del arousal en el $7 \%$.

Finalmente, los trastornos del sueño identificados en el niño y el adolescente en aislamiento social por la pandemia por COVID-19, en orden presentación fueron: el trastorno de inicio y mantenimiento del sueño en el $37 \%$ de los casos, la hiperhidrosis del sueño en el $20 \%$, somnolencia diurna excesiva en el $17 \%$, trastornos en la transición sueño- vigilia en el $14 \%$, trastornos respiratorios en el $7 \%$, arousal en el 2.8\% (Tabla 2). 
Tabla 2. Trastornos del sueño identificados mediante la aplicación la Escala de Trastornos del Sueño para Niños de Bruni en el niño y adolescente en aislamiento social durante la pandemia COVID-19, Hospital General del Norte de Guayaquil, IESS, Los Ceibos, 2021.

\begin{tabular}{lcccccc} 
Grupo & I-M & R & A & T & S & H \\
\hline Preescolar & $7(43 \%)$ & $1(6 \%)$ & 0 & $5(31 \%)$ & $2(12 \%)$ & $5(31 \%)$ \\
\hline Escolar & $13(52 \%)$ & $3(12 \%)$ & $1(4 \%)$ & $5(20 \%)$ & $6(24 \%)$ & $7(28 \%)$ \\
Adolescente & $6(42 \%)$ & $1(7 \%)$ & $1(7 \%)$ & 0 & $4(28 \%)$ & $2(14 \%)$ \\
\hline TOTAL & $26(37 \%)$ & $5(7 \%)$ & $2(2.8 \%)$ & $10(14 \%)$ & $12(17 \%)$ & $14(20 \%)$ \\
\hline
\end{tabular}

I-M: trastornos de inicio y mantenimiento. R: trastornos respiratorios. A: Trastornos del arousal. T: trastornos en la transición sueño-vigilia. S: somnolencia. H: Hiperhidrosis. Elaboración: Cruz D. 2021. Fuente: Formulario de recolección de datos.

\section{DISCUSIÓN}

Durante la actual pandemia de COVID-19, los problemas psicológicos como ansiedad, depresión, irritabilidad, cambios de humor, falta de atención y trastornos del sueño son bastante comunes entre los niños (10).

En el presente estudio el $35 \%$ de los niños $y$ adolescentes en aislamiento social por la pandemia por COVID-19 tuvieron algún trastorno del sueño, otros estudios similares han reportado una prevalencia entre el 25 y $42 \%$ (2). La edad promedio de presentación de trastornos del sueño en niños y adolescentes en aislamiento social por la pandemia por COVID-19 fue de ocho años, el valor de la moda fue de siete años, predominando en el género masculino.

Los trastornos del sueño identificados en el presente estudio fueron: trastornos en el inicio y mantenimiento del sueño, hiperhidrosis del sueño, somnolencia diurna excesiva, trastorno en la transición sueño-vigilia, problemas respiratorios, y arousal. En el 2018 en un estudio de igual características poblacional se reportó: trastornos en la transición sueño-vigilia $38 \%$, problemas respiratorios $18 \%$, problemas en el inicio y mantenimiento del sueño $17 \%$, hiperhidrosis del sueño $15 \%$, problemas del arousal $13 \%$ y somnolencia diurna excesiva en el $10 \%(11,12)$. Es interesante conocer que en estudios poblacionales similares, los datos en relación a trastornos del sueño en niños pre pandemia por COVID-19, no coinciden con los reportados en el presente estudio. Es necesario cuestionarse si el aislamiento social por la pandemia por COVID-19 está ocasionando un mayor número de trastornos del sueño en el inicio o mantenimiento del sueño, o somnolencia diurna.

En referencia a los trastornos del sueño del inicio y mantenimiento del mismo, se reporta una prevalencia entre el 20 a 30\% en lactantes, niños pequeños y en adolescentes del 3 a 12\% (13). En el presente estudio se reportó en el 52\% de escolares, $43 \%$ de preescolares y $42 \%$ de adolescentes. Por ende, en nuestra población de niños y adolescentes en aislamiento social durante la pandemia por COVID-19, existe un mayor porcentaje de casos a lo reportado pre pandemia. En adultos sanos en aislamiento social son factores de riesgo para trastornos del sueño del inicio y mantenimiento: los cambios en el estilo de vida, el miedo a contraer la enfermedad, la edad joven, el sexo femenino, los antecedentes de enfermedades mentales y una menor capacidad de afrontamiento al estrés, la poca exposición al sol; en pediatría, la mala adquisición de los hábitos de sueño lo predispone $(1,7)$.

Con respecto a la hiperhidrosis del sueño, estudios pre pandemia reportan una prevalencia variable entre 7 a $12 \%$ de la población. En nuestro estudio los porcentajes fueron mayores (28\% de escolares, $31 \%$ de preescolares y adolescentes). Bruni y cols, sugirieron considerar a la hiperhidrosis del sueño como un trastorno del sueño infantil característico y 
que merece una mayor investigación (8). Son escasos los estudios sobre este trastorno en la literatura médica, por lo cual se desconoce las correlaciones de la sudoración excesiva durante el sueño en los niños. Es probable que el alto porcentaje reportado en nuestro estudio se relaciona en parte con el clima (temperatura ambiente en los meses de febrero y marzo) de la ciudad de Guayaquil y normas de construcción de viviendas (retención de calor), ya que una temperatura óptima ambiental es necesaria para conciliar el sueño (14).

La prevalencia de somnolencia diurna excesiva es del $10 \%$ en niños y del $50 \%$ en adolescentes (13). En nuestro estudio se reportó en el $48 \%$ de los adolescentes y en el $24 \%$ de los escolares. La somnolencia diurna excesiva es más frecuente secundaria a otros trastornos del sueño, como por ejemplo el sueño insuficiente $(2,7)$; puede existir correlación entre el trastorno de inicio y mantenimiento y la somnolencia diurna excesiva reportada.

Si bien en el presente estudio no evaluamos el sueño de los padres o convivientes, en la literatura médica se describe que los retrasos en los horarios de sueño de los adultos relacionados con responsabilidades laborales modificadas y otros factores tienen un impacto significativo en los horarios de sueño de los niños. Esto sugiere que, con un cambio significativo en el horario diario, incluso durante un período de tiempo relativamente corto, el sueño de los niños pequeños puede sufrir un cambio sustancial (4).

El estudio realizado puede ser subjetivo, el estudiar los trastornos del sueño mediante el uso de cuestionarios dirigidos a los padres no es comparable a la objetividad de estudiarlos con polisomnografía, actigrafía, videografía nocturna y el test de latencias múltiples (7); sin embargo, ayuda a concientizar en la población sobre los trastornos del sueño en pediatría en esta época de pandemia, resaltando la necesidad de mejorar la higiene del sueño en el niño, adolescente y adulto.

Es necesario aclarar que los estudios citados en esta investigación, se realizaron en poblaciones no similares en cuanto sus características geográficas, climáticas y étnicas (o raciales); sin embargo, los tomamos como referencia por haberse realizado en el grupo etario similar a nuestra población y nos permiten tener una visión comparativa objeto de nuestro estudio y ante la falta de estudios pre pandemia en nuestra población específica.

\section{CONCLUSIONES}

Los datos presentados en este estudio, si bien no pueden ser extrapolados a la población general, resaltan la importancia de concientizar a la población sobre los trastornos del sueño en los niños y adolescentes. Con la finalidad de ampliar la visión del presente estudio, se plantea la necesidad de realizar investigación sobre las posibles causas fisiopatológicas y psicopatológicas que pudieran contribuir o determinar el aparecimiento de los trastornos del sueño descritos, con la finalidad de ampliar las recomendaciones en la intervención. La higiene del sueño es un recurso inicial válido en los trastornos del sueño en niños y adolescentes.

\section{AGRADECIMIENTOS}

Al área de Pediatría, servicio de consulta externa, Hospital General del Norte de Guayaquil, IESS, Los Ceibos. Al Dr. David Barzallo Núñez, Psiquiatra Infantil, Hospital Docente Ambato por la asesoría técnica en el presente estudio.

\section{Anexo 1.}

Cuestionario

Formulario número __ Fecha No. De Expediente Clínico

\begin{tabular}{ll} 
Variable & Escala \\
\hline Sexo & $\begin{array}{l}0=\text { Masculino } \\
1=\text { Femenino }\end{array}$ \\
\hline $\begin{array}{l}\text { Qué edad tiene su niño/a } \\
\text { actualmente? }\end{array}$ &
\end{tabular}

\section{PREGUNTA CERRADA DE SELECCIÓN}

DEL CASO

¿Su niño ha presentado alteraciones del sueño durante este periodo de aislamiento social por la pandemia $0=\operatorname{si~} 1=$ no COVID-19?

SI LA PREGUNTA ES AFIRMATIVA CONTINUE CON LA APLICACIÓN DE LA ESCALA DE ALTERACIONES DEL SUEÑO DE LA INFANCIA. DR. O. BRUNI.

Elaboración: Cruz D. 


\section{Anexo 2.}

Escala de alteraciones del sueño en la infancia. Dr. O. Bruni

\begin{tabular}{|c|l|c|c|c|c|c|}
\hline & $\mathbf{1}$ & $\mathbf{2}$ & $\mathbf{3}$ & $\mathbf{4}$ & $\mathbf{5}$ \\
\hline $\mathbf{1}$ & $\begin{array}{l}\text { ¿Cuántas horas duerme la mayoría de las } \\
\text { noches? }\end{array}$ & $9-11$ & $8-9$ & $7-8$ & $5-7$ & $<5$ \\
\hline $\mathbf{2}$ & ¿Cuánto tarda en dormirse?(minutos) & $<15$ & $15-30$ & $30-45$ & $45-60$ & $>60$ \\
\hline
\end{tabular}

En la puntuación de las siguientes respuestas valore de esta forma:

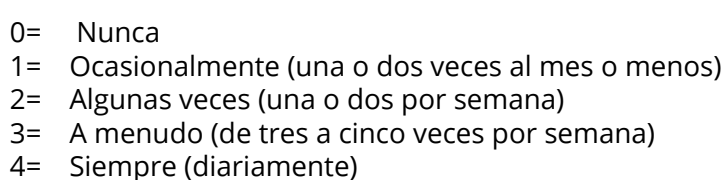

\begin{tabular}{|c|c|c|c|c|c|c|c|c|}
\hline 3 & \multicolumn{3}{|l|}{ Se va a la cama de mal humor } & 0 & 1 & 2 & 3 & 4 \\
\hline 4 & \multicolumn{3}{|c|}{ Tiene dificultad para coger el sueño por la noche } & 0 & 1 & 2 & 3 & 4 \\
\hline 5 & \multicolumn{3}{|c|}{ Parece ansioso o miedoso cuando "cae" dormido } & 0 & 1 & 2 & 3 & 4 \\
\hline 6 & \multicolumn{3}{|c|}{ Sacude o agita partes del cuerpo al dormirse: } & 0 & 1 & 2 & 3 & 4 \\
\hline 7 & \multicolumn{3}{|c|}{$\begin{array}{l}\text { Realiza acciones repetitivas tales como rotación de la cabeza para } \\
\text { dormirse }\end{array}$} & 0 & 1 & 2 & 3 & 4 \\
\hline 8 & \multicolumn{3}{|c|}{ Tiene escenas de "sueños" al dormirse } & 0 & 1 & 2 & 3 & 4 \\
\hline 9 & \multicolumn{3}{|c|}{ Suda excesivamente al dormirse } & 0 & 1 & 2 & 3 & 4 \\
\hline 10 & \multicolumn{3}{|c|}{ Se despierta más de dos veces cada noche } & 0 & 1 & 2 & 3 & 4 \\
\hline 11 & \multicolumn{3}{|c|}{ Después de despertarse por la noche tiene dificultades para dormirse } & 0 & 1 & 2 & 3 & 4 \\
\hline 12 & \multicolumn{3}{|c|}{$\begin{array}{l}\text { Tiene tirones o sacudidas de las piernas mientras duerme, ncambia a } \\
\text { menudo de posición o da "patadas" a la ropa de cama }\end{array}$} & 0 & 1 & 2 & 3 & 4 \\
\hline 13 & \multicolumn{3}{|c|}{ Tiene dificultades para respirar durante la noche } & 0 & 1 & 2 & 3 & 4 \\
\hline 14 & \multicolumn{3}{|c|}{ Da boqueadas para respirar durante el sueño } & 0 & 1 & 2 & 3 & 4 \\
\hline 15 & \multicolumn{3}{|l|}{ Ronca } & 0 & 1 & 2 & 3 & 4 \\
\hline 16 & \multicolumn{3}{|c|}{ Suda excesivamente durante la noche } & 0 & 1 & 2 & 3 & 4 \\
\hline 17 & \multicolumn{3}{|c|}{ Usted ha observado que camina dormido } & 0 & 1 & 2 & 3 & 4 \\
\hline 18 & \multicolumn{3}{|c|}{ Usted ha observado que habla dormido } & 0 & 1 & 2 & 3 & 4 \\
\hline 19 & \multicolumn{3}{|l|}{ Rechina los dientes dormido } & 0 & 1 & 2 & 3 & 4 \\
\hline 20 & \multicolumn{3}{|c|}{$\begin{array}{l}\text { Se despierta con un chillido o confundido de forma que aparentemente } \\
\text { no le reconoce, y no recuerda nada al día siguiente }\end{array}$} & 0 & 1 & 2 & 3 & 4 \\
\hline 21 & \multicolumn{3}{|c|}{ Tiene pesadillas que no recuerda al día siguiente } & 0 & 1 & 2 & 3 & 4 \\
\hline 22 & \multicolumn{3}{|c|}{ Es difícil despertarlo por la mañana } & 0 & 1 & 2 & 3 & 4 \\
\hline 23 & \multicolumn{3}{|c|}{ Al despertarse por la mañana parece cansado } & 0 & 1 & 2 & 3 & 4 \\
\hline 24 & \multicolumn{3}{|c|}{ Parece que no se pueda mover al despertarse por la mañana } & 0 & 1 & 2 & 3 & 4 \\
\hline 25 & \multicolumn{3}{|l|}{ Tiene Soñolencia diurna } & 0 & 1 & 2 & 3 & 4 \\
\hline 26 & \multicolumn{3}{|c|}{ Se duerme de repente en determinadas situaciones } & 0 & 1 & 2 & 3 & 4 \\
\hline & \multicolumn{3}{|r|}{ Total } & & & & & \\
\hline \multicolumn{2}{|c|}{ Factores } & Preguntas & Rango Normal & & \multicolumn{3}{|c|}{ Puntuación } & \\
\hline \multicolumn{2}{|c|}{ Inicio y mantenimiento } & $1-2-3-4-5-10-11$ & $9.9+/-3.11$ & & & & & \\
\hline \multicolumn{2}{|c|}{ Problemas respiratorios } & $13-14-15$ & $3.77+/-1.45$ & & & & & \\
\hline Des & rdenes del arousal & $17-20-21$ & $3.29+/-0.84$ & & & & & \\
\hline $\begin{array}{l}\text { Alte } \\
\text { vigil }\end{array}$ & aciones transición sueño/ & $6-7-8-12-18-19$ & $8.11+/-2.41$ & & & & & \\
\hline Exc & siva somnolencia & $22-23-24-25-26$ & $7.11+/-2.57$ & & & & & \\
\hline Hip & rhidrosis & $9-16$ & $2.87+/-1.69$ & & & & & \\
\hline
\end{tabular}

Punto de corte del trastorno global del sueño: 39. Elaboración: Dr. O. Bruni. 


\section{REFERENCIAS BIBLIOGRÁFICAS}

1. Medina-Ortiz O, Araque-Castellanos F, RuizDominguez LC, Riaño-Garzón $M$, Bermudez V. Trastornos del sueño a consecuencia de la pandemia por COVID-19. Rev Peru Med Exp Salud Pública [Internet]. 2020;37:75561. Disponible en: http://www.scielo.org.pe/ scielo.php?script=sci_arttext\&pid=S172646342020000400755\&nrm=iso

2. Baptista AS, Prado IM, Perazzo MF, Pinho T, Paiva SM, Pordeus IA, et al. Can children's oral hygiene and sleep routines be compromised during the COVID-19 pandemic? Int J Paediatr Dent. 2021;31(1):12-9. DOI: 10.1111/ipd.12732

3. Ramírez-Ortiz J, Fontecha-Hernández J, Escobar-Córdoba F. Efectos del aislamiento social en el sueño durante la pandemia COVID-19. 2019;57(6(36)):23-30. DOI: 10.1590/ SciELOPreprints.801

4. Liu Z, Tang H, Jin Q, Wang G, Yang Z, Chen H, et al. Sleep of preschoolers during the coronavirus disease 2019 (COVID-19) outbreak. J Sleep Res. 2021;30(1):e13142. DOI: 10.1111/jsr.13142

5. Hartley S, Colas des Francs C, Aussert F, Martinot $C$, Dagneaux S, Londe $V$, et al. The effects of quarantine for SARS-CoV-2 on sleep: An online survey TT - Les effets de confinement SARS-CoV-2 sur le sommeil: enquête en ligne au cours de la quatrième semaine de confinement. Encephale [Internet]. 2020/05/11. 2020;46(3S):S53-9. DOI: 10.1016/j.encep.2020.05.003

6. Rana M, Allende C, Latorre T, Astorga K, Torres A. Sueño en los niños: fisiología y actualización de los últimos conocimientos. Med (B Aires). 2019;79(Suppl 3):25-8.

7. Pedemonte V, Gandaro P, Scavone C. Trastornos del sueño en una población de niños sanos de Montevideo: Primer estudio descriptivo. Arch Pediatr Urug [Internet]. 2014;85(1):4-8. Disponible en: http://www.scielo.edu.uy/ scielo.php?script=sci_arttext\&pid=S1688$12492014000100002 \& n r m=$ iso

8. Bruni O, Ottaviano S, Guidetti V, Romoli M, Innocenzi M, Cortesi F, et al. The Sleep Disturbance Scale for Children (SDSC). Construction and validation of an instrument to evaluate sleep disturbances in childhood and adolescence. J Sleep Res. 1996;5(4):251-61. DOI: 10.1111/j.13652869.1996.00251.x

9. Romeo DM, Bruni O, Brogna C, Ferri R, Galluccio $\mathrm{C}$, De Clemente $\mathrm{V}$, et al. Application of the sleep disturbance scale for children (SDSC) in preschool age. Eur J Paediatr Neurol. 2013;17(4):374-82. DOI: 10.1016/j.ejpn.2012.12.009

10. Panda PK, Gupta J, Chowdhury SR, Kumar R, Meena AK, Madaan P, et al. Psychological and Behavioral Impact of Lockdown and Quarantine Measures for COVID-19 Pandemic on Children,
Adolescents and Caregivers: A Systematic Review and Meta-Analysis. J Trop Pediatr. 2021;67(1). DOI: 10.1093/tropej/fmaa122

11. Valderrama E, Herrero C. Trastornos del sueño en la infancia. Clasificación, diagnóstico y tratamiento. An Pediatría Contin. 2014;12(4):17582.

12. Álvarez M, Ledesma J. ¿Cómo duermen nuestros niños? Análisis de los trastornos del sueño en niños. Rev Pediatr Aten Primaria. 2018;20(80):36570.

13. González Rabelino G, Scavone Mauro C. Sueño en pediatría. En: Sueño en pediatría. 1a ed. Montevideo: Ediciones Journal; 2018. p. 24.

14. Simola P. Sleep problems and their implications from preschool to school age [Internet]. University of Helsinki; 2014. Disponible en: https://helda. helsinki.fi/handle/10138/45386

\section{ACERCA DE LOS AUTORES}

1. Daniel Leonardo Cruz Montesinos. Servicio de Neurología Pediátrica, Hospital General del Norte de Guayaquil, IESS, Los Ceibos, Guayaquil-Ecuador.

\section{ORCID: 0000-0002-6417-9164}

2. María Andrea Linares Contreras. Servicio de Neurología Pediátrica, Hospital General del Norte de Guayaquil, IESS, Los Ceibos, Guayaquil-Ecuador.

ORCID: 0000-0002-4041-5839

3. Mignia Ziadet Bermúdez. Servicio de Pediatría. Hospital General del Norte de Guayaquil, IESS, Los Ceibos, GuayaquilEcuador.

\section{ORCID: 0000-0002-7365-6436}

4. Janiny Ortiz Luna. Servicio de Pediatría. Hospital General del Norte de Guayaquil, IESS, Los Ceibos, Guayaquil-Ecuador.

ORCID: 0000-0001-7755-1095

5. Francisco Morán Rizzo. Servicio de Pediatría. Hospital General del Norte de Guayaquil, IESS, Los Ceibos, GuayaquilEcuador.

\section{ORCID: 0000-0002-1636-6299}

6. Nathaly Baldeón Estrada. Servicio de Pediatría. Hospital General del Norte de Guayaquil, IESS, Los Ceibos, GuayaquilEcuador.

ORCID: 0000-0003-2108-6221 